ARTIGOS

Submetido em 24.07.2012. Aprovado em 01.11.2013

Avaliado pelo sistema double blind review. Editora Científica: Mariangela Leal Cherchiglia

http://dx.doi.org/10.1590/So034-759020140409

\section{VALORES RELATIVOS AO TRABALHO DE PESQUISADORES EM UMA ORGANIZAÇÃO BRASILEIRA}

\author{
Work values of researchers in a Brazilian organization \\ Valores relativos al trabajo de investigadores en una empresa brasileña
}

\section{RESUMO}

O objetivo desta pesquisa foi analisar os valores relativos ao trabalho de pesquisadores em uma empresa de P\&D brasileira, definindo-se suas prioridades axiológicas laborais. Estudos têm evidenciado lacunas sobre gestão de pesquisadores em organizações inovadoras, sendo esse recurso humano envolvido diretamente com a inovação. Por meio da compreensão dos valores pessoais laborais de pesquisadores em empresa de P\&D, torna-se possivel avaliar o que esses profissionais consideram importante em seu ambiente de trabalho, subsidiando conhecimento aos gestores de trabalhadores do conhecimento e possibilitando melhorias organizacionais. Por meio de um questionário validado, 208 pesquisadores da firma de P\&D brasileira participaram deste estudo. Os resultados possibilitaram a compreensão da organização dos valores do trabalho dos pesquisadores, bem como suas prioridades axiológicas laborais, sendo estas definidas pela seguinte sequência: relações sociais, realização profissional, estabilidade e prestígio. Implicações e limitações do estudo foram levantadas e discutidas.

PALAVRAS-CHAVE | Valores relativos ao trabalho, pesquisadores, organização de pesquisa e desenvolvimento, prioridades axiológicas laborais, survey.

\begin{abstract}
The objective of this paper was to analyze values related to researcher work at a Brazilian R\&D company, defining their axiological work priorities. Studies have evidenced gaps in researcher management at innovative organizations, with this human resource directly involved in innovation. Through an understanding of researchers' personal work values at an R\&D company, it becomes possible to evaluate what these professionals consider important subsidizing knowledge to managers of knowledge workers and enabling organizational improvements in their work environment. By using a valid questionnaire, 208 researchers at a Brazilian $R \& D$ firm participated in this study. The results made it possible for the organization to understand the researchers' work values, as well as their axiological work priorities defined according to the following sequence: social relations, professional realization, stability and prestige. Study implications and limitations were raised and discussed.
\end{abstract}

KEY WORDS / Work-related values, researchers, research and development organization, axiological work priorities, survey.

Doutoranda em Psicologia pela Faculdade de Filosofia, Ciências e Letras de Ribeirão Preto, Universidade de São PauloRibeirão Preto - SP, Brasil

FERNANDO CÉSAR ALMADA SANTOS almada@sc.usp.br

Professor do Departamento de Engenharia de Produção, Universidade de São Paulo - São Carlos - SP, Brasil

FRANCISCO ANTÔNIO ROJAS ROJAS franciscoantoniorojas@hotmail.com Professor do Centro de Ciências Exatas e de Tecnologia, Universidade Federal de São Carlos - São Carlos - SP, Brasil

\section{RESUMEN}

El objetivo de este estudio fue analizar los valores relativos al trabajo de los investigadores en una empresa de I\&D brasileña, definiéndose sus prioridades axiológicas laborales. Los estudios han evidenciado lagunas sobre la gestión de investigadores en organizaciones innovadoras, siendo este recurso humano implicado directamente con la innovación. Por medio de la comprensión de los valores personales y laborales de investigadores en empresas de I\&D, es posible evaluar lo que estos profesionales consideran importante en su ambiente de trabajo, proporcionando conocimiento a los gestores de trabajadores del conocimiento y posibilitando mejoras organizacionales. Por medio de un cuestionario validado, 208 investigadores de la firma de I\&D brasileña participaron de este estudio. Los resultados posibilitaron la comprensión por la organización de los valores del trabajo de los investigadores, así como también sus prioridades axiológicas laborales, siendo estas definidas por la siguiente secuencia: relaciones sociales, realización profesional, estabilidad y prestigio. Implicancias y limitaciones del estudio fueron relevadas y discutidas.

PALABRAS CLAVE / Valores relativos al trabajo, investigadores, organización de investigación y desarrollo, prioridades axiológicas laborales, survey. 


\section{INTRODUÇÃO}

Pesquisas evidenciam que os executivos têm, cada vez mais, declarado que as despesas com recursos humanos são vistas como investimento estratégico e que, embora as pessoas sempre tenham sido fundamentais nas empresas, atualmente elas desempenham um papel ainda mais central para a obtenção de vantagem competitiva. Para Drucker (1999), as pessoas de bom desempenho são os recursos mais escassos da organização atual, e, portanto, é necessário que gerentes aloquem recursos humanos com os mesmos cuidados que fazem com o capital. 0 sucesso da organização depende cada vez mais das competências e motivação de seus empregados e de sua gestão (Bohlander, Snell, \& Sherman, 2003).

Dessa forma, o alcance de bons resultados organizacionais está diretamente relacionado ao desempenho e produtividade dos recursos humanos que compõem a organização, e, para isso, faz-se necessário que as pessoas sejam mobilizadas a atingir os objetivos propostos pela empresa. É fundamental, assim, que os gestores compreendam o que seus funcionários valorizam no ambiente de trabalho e quais variáveis interferem em seu comportamento organizacional. Estudos sobre valores no contexto laboral passam a ser um dos focos importantes de pesquisas na área de gestão de pessoas, visando à compreensão sobre o que é importante para os indivíduos no ambiente de trabalho, enfatizando o entendimento dos motivos pelos quais as pessoas executam suas tarefas laborais (Porto \& Tamayo, 2008).

Em pesquisas atuais, estudiosos propuseram um modelo que explicasse a estrutura de valores humanos em diversos contextos, como trabalho, religião, família, entre outros (Schwartz, 1992, 1999, 2011; Tamayo, 2007a), sendo o contexto laboral o que tem recebido maior ênfase. Com base no estudo de valores pessoais relativos ao trabalho, avanços consideráveis foram feitos na compreensão sobre o que motiva seres humanos em seus ambientes laborais, possibilitando aos gestores a identificação de metas consideradas importantes pelos seus funcionários (Porto \& Tamayo, 2003, 2007a, 2007b, 2008; Ros, Schwartz, \& Surkiss, 1999; Tamayo \& Paschoal, 2003). A priorização de determinados valores do trabalho é definida como prioridade axiológica laboral. Sua compreensão e conhecimento podem "subsidiar o desenvolvimento de estratégias organizacionais que auxiliem na promoção do bem-estar dos indivíduos, bem como na melhoria dos resultados organizacionais por meio de funcionários mais motivados para o trabalho" (Porto \& Tamayo, 2008, p. 296).

Visto que os valores podem variar entre organizações com culturas específicas e contextos de atuação, e entre tipos ocupacionais (Tamayo, Faria, Filho, Tavares, Carvalho, \& Bertolinni, 1998), estudos que focam os valores pessoais laborais de tra- balhadores de determinada área têm se mostrado relevantes, já que tais particularidades podem trazer implicações nas práticas de gestão (Lundberg, Gudmundson, \& Andersson, 2009).

Organizações com ambientes inovadores, que lidam com ciência, tecnologia e inovação ( $C, T$ \& I), e pesquisa e desenvolvimento (P\&D), possuem algumas características peculiares importantes no que diz respeito à gestão de pessoas (Coda \& Ricco, 2010; Jordan, 2005; Katz, 2005). São consideradas organizações inovadoras as instituições de P\&D, que se dedicam fundamentalmente a tais atividades, sendo dessa natureza os centros tecnológicos de indústrias, laboratórios, centros em universidades e organizações estatais exclusivas de P\&D (Verdu \& Bulgacov, 2001). Segundo Jordan (2005), lacunas existentes em dados empíricos disponíveis sobre a tarefa de gestão de pesquisadores em instituições de P\&D ainda motivam gestores de ambientes inovadores a tentar compreender o que constitui um bom ambiente de pesquisa e quais ferramentas podem ser utilizadas para a gestão desses profissionais. Gestores de organizações dessa natureza requerem também ajuda no que diz respeito a atrair, motivar e reter pesquisadores (Jordan, 2005), e estudos que objetivam conhecer mais sobre a gestão desses profissionais ainda estão em seu início (Davenport, 2005).

É importante ressaltar que a inovação tem sido discutida como uma das estratégias prioritárias para adquirir vantagem competitiva no mercado atual, sendo um pré-requisito para o sucesso e sobrevivência de uma organização, e até mesmo de uma sociedade (Jamrog, Vickers, \& Bear, 2006). Sendo os pesquisadores os recursos humanos envolvidos diretamente com a inovação, enfatiza-se, assim, seu papel fundamental nos avanços sociais e no contexto nacional, portanto tal profissional merece o foco de pesquisas.

Segundo Berson and Linton (2005), a liderança efetiva de profissionais de $P \& D$, bem como a qualidade da gestão desse recurso humano, é a chave para os resultados de sucesso. É necessário compreender as variáveis que interferem no comportamento dos pesquisadores, a fim de proporcionar uma boa gestão, e possivelmente responder a perguntas relacionadas ao planejamento e à organização do trabalho em empresas inovadoras de P\&D.

Dessa forma, os valores laborais mostram-se variáveis importantes que merecem investigação nesse contexto, ou seja, focando os recursos humanos de organizações inovadoras, considerando-se as particularidades desse âmbito. Por meio da compreensão desse fenômeno, é possível repensar estratégias utilizadas na gestão de pesquisadores, comparando os dados encontrados em pesquisa com a realidade prática de instituições inovadoras e com a literatura, e obtendo-se novas informações e possíveis ferramentas que contribuam com a gestão motivacional da equipe. 
A presente pesquisa teve, assim, o objetivo de analisar a organização dos valores relativos ao trabalho de pesquisadores em uma organização de P\&D brasileira, definindo-se as prioridades axiológicas laborais da amostra estudada.

\section{TEORIA SOBRE VALORES RELATIVOS AO TRABALHO}

Os valores pessoais laborais têm sido discutidos por pesquisadores há décadas, sendo importantes variáveis que interferem nas ferramentas de gestão e no comportamento organizacional. Seu estudo visa compreender o que é importante para as pessoas no ambiente laboral, investigando-se quais motivos levam as pessoas a trabalhar (Jin \& Rounds, 2012; Paarlberg \& Perry, 2007; Porto \& Tamayo, 2008). Algumas teorias foram propostas a fim de sistematizar o conhecimento sobre os valores relativos ao trabalho, chegando-se a definições interessantes utilizadas em pesquisas empíricas.

A teoria de Super (1953) apresenta alguns motivos que levam as pessoas a gostar ou não de sua ocupação laboral, e o autor realizou revisão da literatura na época para chegar a suas conclusões. Propôs três necessidades que podem ser satisfeitas no trabalho: as necessidades de relações humanas, de trabalho e de sustento. Embora seja ainda citada na literatura sobre valo- res laborais, a teoria de Super (1953) não apresenta uma definição consistente sobre esse conceito, confundindo-o com o conceito de satisfação no trabalho (Porto \& Tamayo, 2008).

Elizur and Sagie (1999) também são autores de importantes estudos sobre valores laborais, propondo teorias e definições. Para eles, os valores laborais são os fatores desejados pelas pessoas no trabalho. São as metas ou recompensas que as pessoas buscam por meio do trabalho (Sagie \& Elizur, 1996). Esses estudiosos sugerem, ainda, que os valores laborais podem ser investigados por meio de facetas, definidas como um critério ou regra para classificar itens associados com um dado conceito, nesse caso, os valores pessoais no ambiente de trabalho.

Por meio da teoria de valores pessoais de Schwartz (1992), estudos no âmbito do trabalho foram realizados na tentativa de verificar se a teoria geral de valores humanos também se aplicava ao contexto específico laboral. Schwartz (1992), em pesquisa empírica realizada em vários países, investigou a organização dos valores humanos, propondo uma estrutura universal composta por 10 tipos motivacionais, denominados: poder, realização, hedonismo, estimulação, autodeterminação, universalismo, benevolência, tradição, conformidade e segurança. Cada um deles representa uma meta ou motivação e expressa interesses, sendo estes individualistas, coletivistas ou mistos, representados no Quadro 1.

\section{Quadro 1. Relação entre tipos motivacionais, valores pessoais, suas metas e interesses}

\begin{tabular}{|c|c|c|c|}
\hline Tipos motivacionais & Valores pessoais envolvidos & Metas & Interesse \\
\hline Realização & $\begin{array}{l}\text { Ambição, influência e } \\
\text { inteligência }\end{array}$ & $\begin{array}{l}\text { Sucesso pessoal obtido por meio de uma demonstração de competência, } \\
\text { influência sobre os outros, realização pessoal e profissional }\end{array}$ & Individual \\
\hline Poder & $\begin{array}{l}\text { Poder social, autoridade e } \\
\text { riquezas }\end{array}$ & Controle sobre pessoas e recursos, prestígio e status social & Individual \\
\hline Estimulação & Audácia & $\begin{array}{l}\text { Excitação, novidade, mudança, desafios, explorar, inovar, adquirir novos } \\
\text { conhecimentos }\end{array}$ & Individual \\
\hline Conformidade & Polidez e autodisciplina & $\begin{array}{l}\text { Controle de impulsos e ações que podem transgredir normas e expectativas } \\
\text { sociais ou que podem prejudicar os outros }\end{array}$ & Coletivo \\
\hline Segurança & $\begin{array}{l}\text { Ordem social e segurança } \\
\text { familiar }\end{array}$ & $\begin{array}{l}\text { Integridade pessoal, harmonia e estabilidade da sociedade, do } \\
\text { relacionamento e de si mesmo }\end{array}$ & Misto \\
\hline Universalismo & $\begin{array}{l}\text { Igualdade e proteção da } \\
\text { natureza e meio ambiente }\end{array}$ & $\begin{array}{l}\text { Tolerância, compreensão e promoção do bem-estar de todos na sociedade } \\
\text { e grupo, e proteção da natureza }\end{array}$ & Misto \\
\hline
\end{tabular}

Fonte: Adaptado de Tamayo e Paschoal (2003) e Demo (2008). 
O estudioso propôs que tais tipos motivacionais de valores estão organizados em um modelo circular e dinâmico, representado pela Figura 1, e os tipos adjacentes possuem compatibilidade, a qual, por meio do continuum circular, vai diminuindo gradualmente até encontrar o tipo motivacional antagônico, em que há maior conflito (Schwartz, 1992, 1999), formando-se, assim, polaridades.

\section{Figura 1. Modelo teórico das relações entre os 10 tipos motivacionais e suas polaridades}

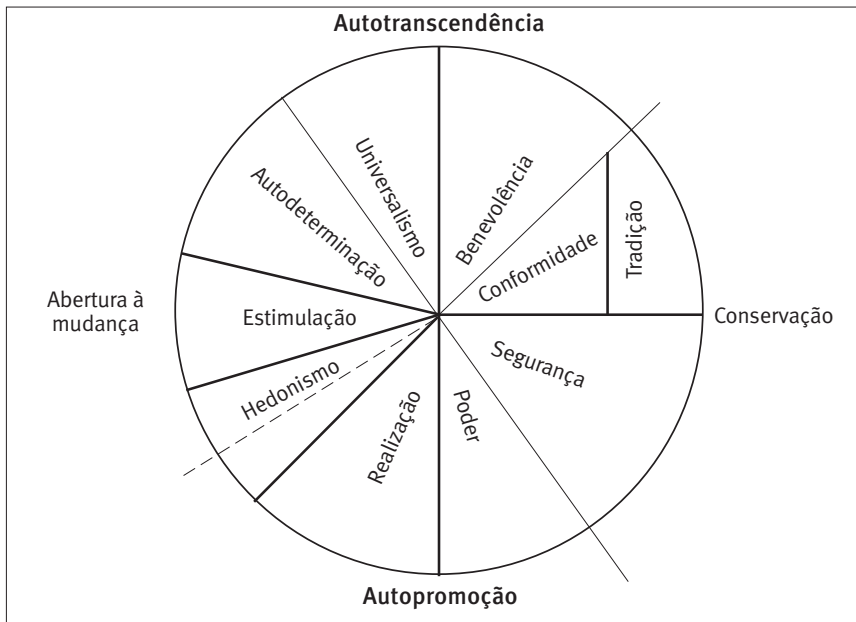

Fonte: Adaptado de Schwartz (1992).

Dessa forma, os 10 tipos motivacionais podem ser agrupados em conjuntos motivacionais fundamentais ou em fatores motivacionais de segunda ordem, que representam as quatro polaridades da estrutura motivacional, sendo esta, portanto, uma estrutura bidimensional. São elas: abertura à mudança versus conservação, e autotranscendência versus autopromoção, cada uma contendo certos valores (Schwartz, 2005).

Considerando a teoria de valores de Schwartz (1992), Ros et al. (1999) encontraram compatibilidade entre três agrupamentos definidos em estudos da literatura da área com os valores de segunda ordem propostos por Schwartz (1999, 2005), sendo eles: valores intrínsecos, que estariam associados à abertura à mudança; valores extrínsecos, que estariam associados à conservação, e, por fim, valores sociais ou afetivos, que estariam associados à autotranscendência. É importante salientar que os modelos já não consideravam a dicotomia intrínseco versus extrínseco, proposta por teóricos como Herzberg (1971), pois apenas essas duas categorias não são suficientes para englobar todos os valores laborais.

Apesar de os achados mostrarem semelhanças entre três grupos de valores e os conjuntos motivacionais de segunda ordem, Ros et al. (1999) consideraram que acrescentar o tipo prestígio poderia tornar o modelo mais completo e suprir lacunas, adicionan- do, então, o quarto grupo, que corresponderia à autopromoção. Esses autores definiram, assim, quatro conjuntos de valores relativos ao trabalho, com base na teoria de valores humanos gerais:

- Intrínsecos, que se referem a metas obtidas pelo conteúdo do próprio trabalho;

- $\quad$ Extrínsecos, que se referem a metas obtidas pelos resultados do trabalho;

- $\quad$ Sociais, que se referem à busca de metas relacionais; e

- Valores de prestígio, que se referem à busca de poder e prestígio por meio do trabalho.

Conforme o modelo proposto, os valores extrínsecos estão em oposição aos intrínsecos, e valores sociais, em oposição aos de prestígio.

No Brasil, Porto e Tamayo (2003) realizaram estudos baseados na teoria de Ros et al. (1999). Esses autores propõem uma definição mais abrangente sobre os valores relativos ao trabalho, que será utilizada no presente estudo. Valores relativos ao trabalho são, assim:

[...] princípios ou crenças sobre metas ou recompensas desejáveis, hierarquicamente organizados, que as pessoas buscam por meio do trabalho e que guiam as suas avaliações sobre os resultados e contexto do trabalho, bem como o seu comportamento no trabalho e a escolha de alternativas de trabalho (Porto \& Tamayo, 2003, p. 146).

Tais autores consideram três aspectos para a definição desse conceito. 0 primeiro é o aspecto cognitivo, que infere que os valores relativos ao trabalho são crenças do que é ou não desejável no trabalho; o segundo é o aspecto motivacional, que considera que os valores laborais expressam interesses e desejos do indivíduo em relação a seu trabalho, e, por fim, há o aspecto hierárquico, isto é, os valores do trabalho são avaliados ao longo de um continuum de importância.

Porto e Tamayo (2003) encontraram em sua pesquisa quatro fatores de segunda ordem que englobam os valores relativos ao trabalho. Tais fatores encontrados foram denominados (Porto \& Tamayo, 2007; Tamayo \& Paschoal, 2003):

- Realização profissional, que se refere à busca de realização pessoal e profissional, e de independência de pensamento e ação no trabalho por meio da autonomia intelectual e da criatividade. É também definido como polo da procura de mudança;

- Estabilidade, que se refere à busca de segurança e ordem na vida pessoal e profissional por meio do trabalho, e a preservação do status quo, possibilitan- 
do suprir materialmente as necessidades pessoais. $\hat{E}$ também definido como polo da estabilidade;

- $\quad$ Relações sociais, que se refere à busca de relações sociais positivas no trabalho e de contribuição positiva para a sociedade por meio do trabalho realizado. É também definido como polo da motivação altruística; e
- $\quad$ Prestígio, que se refere à busca de autoridade, sucesso profissional e poder de influência no ambiente de trabalho. É também definido como polo do prestígio.

A Figura 2 ilustra os conjuntos fundamentais, suas polaridades e seus valores laborais associados. É possível observar que os polos mostram as duas dimensões que englobam os conjuntos motivacionais fundamentais.

Figura 2. Polaridades dos valores relativos ao trabalho de ordem superior

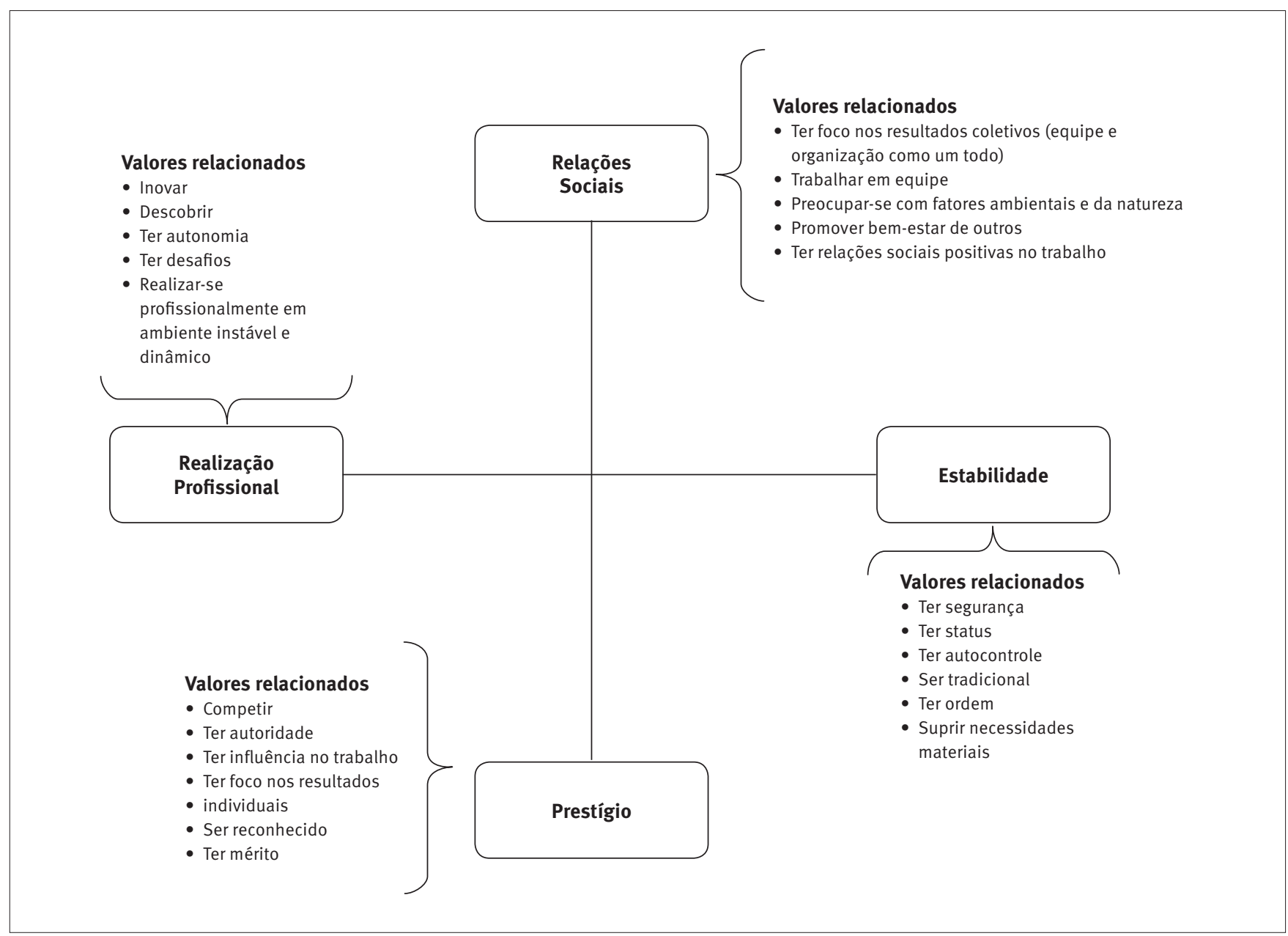

Fonte: Adaptado de Porto e Tamayo (2003).

A primeira dimensão bipolar é constituída pela realização profissional versus estabilidade. Em um polo, é possível encontrar valores relacionados à motivação para inovar, criar, descobrir, autodeterminar-se, ter autonomia e ter desafios na vida e no trabalho. Refere-se ao interesse da pessoa em seguir caminhos incertos e ambíguos para realização profissional e pessoal. Em oposição, encontra-se a procura pela estabilidade e preservação da segurança e status nas relações com os colegas de trabalho e instituições. Refere-se ao interesse da pessoa em procurar segurança, ordem social, autocontrole e respeito à tradição.
Já a segunda dimensão bipolar é composta pelos polos relações sociais versus prestígio. Em um polo, estão os valores para transcender preocupações egoístas e promover o bem -estar dos outros e da natureza. São os valores do empregado relacionados aos resultados de sua equipe, de sua área e da organização como um todo. No polo oposto, estão os valores relacionados ao alcance de interesses próprios do trabalhador e à competição com os outros membros da organização, para ter prestígio, poder. influência e destaque, sem preocupação com o coletivo (Porto \& Tamayo, 2003; Tamayo \& Paschoal, 2003). 
Tamayo e Paschoal (2003) definem que a estrutura motivacional de um indivíduo seja composta pela interação dinâmica entre os quatro conjuntos fundamentais dos valores relativos ao trabalho. Além disso, pela teoria, os conjuntos são organizados por meio de uma hierarquia, em que um ou mais conjuntos tendem a se destacar, conforme os valores pessoais daquele empregado ou grupo de empregados, definindo-se suas prioridades axiológicas laborais: um perfil motivacional.

\section{PESQUISADOR EM ORGANIZAÇÃO DE P\&D}

Considerando as principais características das organizações inovadoras, pode-se enfatizar que a mais importante refere-se à busca por vantagem competitiva no mercado por meio da inovação e de especificidades de seu ambiente organizacional. É fato que a inovação na organização de P\&D não ocorre de modo acidental, mas, sim, porque gestores procuram liderar seus recursos humanos, isto é, os pesquisadores ou trabalhadores do conhecimento, de maneira efetiva para criar e inovar produtos e serviços (Gupta \& Singhal, 1993). Algumas pesquisas foram, assim, direcionadas por perguntas relacionadas aos fatores que motivam os pesquisadores a produzir e o que é considerado importante por esse recurso humano.

Segundo Jordan (2005), a maioria das pesquisas sobre atitudes, valores e satisfação dos pesquisadores não contempla características específicas que cientistas e engenheiros consideram ser fundamentais para organizações de P\&D. Jordan (2005) cita que esses estudos não levam em conta, por exemplo, o fato de que pesquisadores trabalham menos por dinheiro e mais por mérito, como os desafios para superar problemas técnicos e de pesquisa, a satisfação em exercer sua criatividade e a aprovação de seu trabalho por seus pares. Além disso, os profissionais em questão também analisam como importante o pensamento crítico, a troca de ideias e a presença de uma forte fundamentação de pesquisa básica. Embora existam tais afirmações, lacunas existentes sobre a gestão de pesquisadores ainda motivam gestores de ambientes de P\&D a compreender o que constitui um bom ambiente de pesquisa e quais ferramentas podem ser utilizadas para sua melhoria.

Katz (2005) também discute que profissionais de P\&D valorizam colocar em prática uma variedade de habilidades e capacidades. Além disso, dimensões como autonomia e feedback também são consideradas importantes e valorizadas pelos cientistas no ambiente inovador, no entanto, a dimensão crucial está relacionada ao significado da tarefa. Os pesquisadores sentem-se energizados quando consideram que estão fazendo algo importante, algo que realmente faça a diferença.
Por meio do levantamento de dados na revisão da literatura, é possível chegar a conclusões, por meio dos elementos comuns realçados como característicos do ambiente inovador e dos fatores que são valorizados pelos pesquisadores (Jordan, 2005; Katz, 2005; Maccoby, 2006; Miguel \& Teixeira, 2009; Pereira, \& Saes, 1997; Porto \& Tamayo, 2003; Silva \& Ricco, 1998; Tamayo \& Paschoal, 2003; Vojak, Griffin, Price, \& Perlov, 2006). Ao se considerarem os valores relativos ao trabalho associados ao profissional em questão, fica clara a hipótese de que os profissionais do conhecimento valorizam primordialmente a possibilidade de descobrir e inovar no trabalho, os desafios e o significado da tarefa, um ambiente com pouca rotina e a autonomia, sendo possivelmente o conjunto de valores denominado realização profissional o predominante no perfil do pesquisador em uma organização de P\&D. É possível observar que alguns valores do conjunto prestígio, como necessidade de obter mérito e reconhecimento, também podem ser característicos do perfil do pesquisador.

A Figura 3 ilustra os principais valores relativos ao trabalho desse profissional e suas características levantadas com base na revisão da literatura.

Estudos encontrados na literatura permitem concluir que, dependendo da ocupação ou classe de trabalhador, é possível haver diferenças importantes nos valores pessoais gerais e nos valores laborais (Hagström \& Kjellberg, 2007; Tamayo et al, 1998). Tamayo et al. (1998), baseando-se na teoria de valores de Schwartz (1992), realizaram uma pesquisa com o objetivo de investigar as prioridades axiológicas de músicos e advogados, sendo possível definir um perfil de cada tipo ocupacional. Músicos tenderam a valorizar fatores relacionados à mudança, ao individualismo e autonomia, já os advogados mostraram valorizar fatores relacionados à estabilidade e ao prestígio.

Segundo Tamayo et al. (1998), existem, assim, elementos que levaram pesquisadores a formular a hipótese da existência de uma relação estreita entre os valores e as opções profissionais, e as prioridades axiológicas do indivíduo revelam o seu perfil motivacional. Como exemplo, Hagström and Kjellberg (2007) investigaram as diferenças existentes entre profissionais enfermeiros e engenheiros de ambos os sexos. Em geral, os enfermeiros valorizaram mais os fatores relacionados ao altruísmo em comparação aos engenheiros.

O presente estudo pretendeu, assim, investigar empiricamente os valores laborais dos pesquisadores em uma organização pública de P\&D brasileira, verificando se há a confirmação de que o fator realização profissional, de fato, engloba os principais valores relativos ao trabalho da amostra estudada. Por meio da investigação da organização dos quatro conjuntos dos valores, é possível compreender as prioridades axiológicas laborais dos pesquisadores. 
Figura 3. Possível conjunto predominante de valores relativos ao trabalho do pesquisador em organização de P\&D

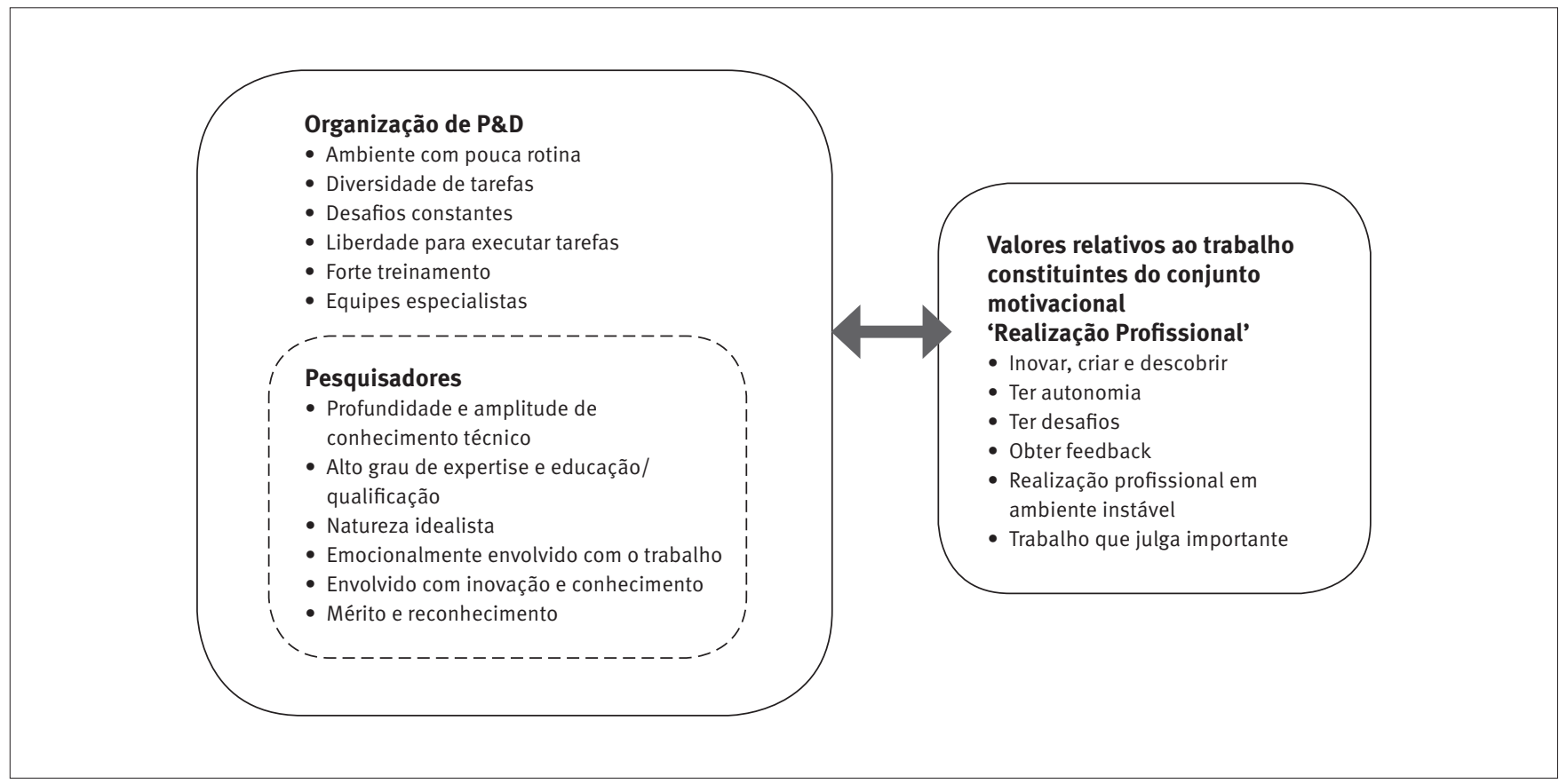

Fonte: Baseado na revisão da literatura.

\section{MÉTODO DE PESQUISA}

O método empregado caracteriza-se pelo processamento de dados quantitativos por meio de pesquisa tipo survey. Esse tipo de estudo envolve a coleta de informações relacionadas a assuntos ligados aos participantes da pesquisa ou às organizações nas quais atuam, e é utilizado para coleta de dados que envolvem grandes amostras (Forza, 2002; Hair Jr, Babin, Money, \& Samouel, 2005). Como a amostra foi retirada de apenas uma organização de P\&D, trata-se da survey de um caso.

\section{Participantes}

Participaram do estudo 208 pesquisadores de uma organização pública de P\&D brasileira, que foram convidados e aceitaram voluntariamente participar dessa pesquisa, sendo uma amostra não probabilística por conveniência. Tais profissionais eram diretamente relacionados à pesquisa e à inovação, caracterizando-se por sua alta qualificação e especialização em áreas de conhecimento.

Os dados sociodemográficos indicaram que 57,7\% ( $n=$ 119) da amostra foram compostos por pessoas do sexo masculino, sendo, portanto, $42,3 \%(n=87)$ pessoas do sexo feminino. A maioria era casada $(68,5 \%)$ e a idade média da amostra foi de 45 anos ( $D P=9,85$ ), variando entre 31 e 73 anos. Com relação à escolaridade, $83,6 \%$ possuíam, ao menos, doutorado comple- to, $1,5 \%$ estavam cursando o pós-doutorado e $21,8 \%$ possuíam pós-doutorado completo. 0 tempo de serviço na empresa mostrou que a maioria, 47,5\%, está há menos de 10 anos na empresa.

\section{Instrumento}

Os dados foram coletados por meio de questionário validado, atualizado e revisado em pesquisas brasileiras (Porto \& Pilati, 2010; Porto \& Tamayo, 2003), denominado Escala Revisada de Valores Relativos ao Trabalho (EVT-R), construído com base na teoria geral de valores de Schwartz (1992) e de Ros et al. (1999).

0 instrumento em questão possui 34 valores do trabalho, ou variáveis, que devem ser analisados pelos respondentes por meio de uma escala Likert de 5 pontos, sendo 1 - Nada importante e 5 - Extremamente importante. A EVT-R é considerada um instrumento adequado para se atingirem os objetivos dessa pesquisa e considerando-se o objeto escolhido nesse estudo. Essa escala apresenta boa validade de construto e permite o avanço da teorização e mensuração dos valores do trabalho, possibilitando a compreensão de como os valores laborais afetam as escolhas e comportamentos no trabalho (Porto \& Pilati, 2010).

Além das questões da EVT-R, o questionário utilizado continha variáveis sociodemográficas dos participantes, como idade, sexo, estado civil, tempo de serviço na empresa e escolaridade. 


\section{Coleta e procedimentos para análise de dados}

Os dados foram coletados com participantes de 14 unidades de uma organização pública de P\&D. Após autorização escrita do responsável de cada unidade, um e-mail convite explicando os objetivos do estudo foi enviado a 770 pesquisadores. Também foi enviado um Termo de Consentimento Livre e Esclarecido, ressaltando-se o caráter voluntário de participação na pesquisa, anonimato e sigilo de informações pessoais. Depois do aceite, os participantes poderiam acessar o questionário completo por meio de um link para responder aos itens em modalidade on-line. No total, obteve-se um retorno de 208 pesquisadores. A coleta de dados teve duração de 27 dias.

A análise dos dados foi realizada por meio de ferramentas estatísticas consideradas as mais adequadas para se atingirem os objetivos da pesquisa. Primeiramente, foi realizada uma análise estatística descritiva dos dados sociodemográficos a fim de se caracterizar a amostra, utilizando-se o programa Microsoft Excel 2007. Em seguida, foram analisadas as respostas de cada participante, a fim de verificar a distribuição das respostas na escala Likert. Optou-se por eliminar os respondentes que escolheram o mesmo número da escala em mais de $80 \%$ dos itens, objetivando-se diminuir a possibilidade de participação não interessada ou de leitura pouca atenta às instruções do questionário. Com base nesse critério, apenas dois respondentes foram eliminados, sendo a amostra final composta por 206 participantes.

Optou-se por realizar a análise fatorial dos dados coletados, considerando-se apenas as variáveis da EVT-R. Para isso, utilizou-se o programa estatístico Statistical Package for the Social Sciences (SPSS) v.17. Segundo Hair et al. (2005), para aplicação da análise fatorial, o pesquisador deve ter um tamanho mínimo de amostra de cinco vezes o número de variáveis analisadas. A amostra composta por 206 participantes é, assim, considerada adequada para essa análise. Como o envio do questionário apenas era possível se todas as questões fossem respondidas, não houve casos omissos.

A análise fatorial (componentes principais) dos dados foi realizada com rotação promax ou oblíqua, visto que, pela teoria utilizada, os componentes ou fatores podem estar correlacionados entre si, e, segundo Hair et al. (2005), esse tipo de rotação é o mais adequado nessas situações. Foi realizado também o cálculo de coeficientes de medida de adequação da amostra - coeficiente de Kaiser-Meyer-Olkin (KMO) - e matriz anti-imagem. Além disso, foram realizadas análises de confiabilidade por meio do cálculo do Alfa de Cronbach (a) dos agrupamentos encontrados.

Posteriormente, com o objetivo de se analisarem as prioridades axiológicas laborais da amostra estudada, foram calculadas as médias dos fatores agrupados, denominados, nesse caso, tipos motivacionais, sendo organizados de modo decrescente: do fator considerado mais importante para o fator considerado menos importante, bem como as médias dos conjuntos motivacionais de segunda ordem, conforme a teoria utilizada.

\section{RESULTADOS}

Os resultados das análises de fatorabilidade da matriz indicaram sua adequação para o uso da análise fatorial. 0 KMO foi de o,81 e o teste de esfericidade de Barlett foi significativo (o,0oo), o que indica boa adequação de tamanho da amostra Hair et al. (2005). Inicialmente, foram encontrados nove fatores na análise. Ao se realizarem os cálculos dos índices de confiabilidade de coerência interna (a) dos itens agrupados, no entanto, verificou-se que o nono fator, composto por apenas dois itens, apresentava intensidade de associação muito abaixo de o,6 Hair et al. (2005). Optou-se, assim, por nova análise fatorial com rotação promax e extração de oito fatores para verificar agrupamento desses itens.

Para essa análise final, a variância total explicada foi de $63,54 \%$, considerada satisfatória Hair et al. (2005). Analisando-se a linha diagonal principal da matriz anti-imagem, observou-se que grande parte dos itens apresentou coeficientes superiores a o,8, sendo estes considerados elevados Hair et al. (2005). 0 restante da matriz revelou valores menores do que 0,3, mostrando apenas 10 valores acima desse número em toda a matriz, que possuía um total de 561 coeficientes de correlação. Entre esses 10 números acima de 0,3, a maior correlação encontrada foi de o,6, para os itens 8 - Obedecer às normas do trabalho e 10 - Respeitar a hierarquia. Optou-se, no entanto, por manter ambos os itens nas análises, podendo esse valor ser considerado correspondente à força de associação moderada Hair et al. (2005). Os resultados encontrados na análise fatorial podem ser visualizados na Tabela 1.

Os índices de confiabilidade de coerência interna (a) foram considerados muito bons para os fatores 1, 2, 3 e 4, bons para os fatores 5 e 7, e moderados para os fatores 6 e 8 Hair et al. (2005). A correlação item-total dentro dos fatores foi superior a 0,3 para todos os itens, assim como descrito pelo estudo de validação realizado pelos autores da escala (Porto \& Pilati, 2010).

Observa-se que as cargas fatoriais foram superiores a o,3 para todos os itens que compõem os agrupamentos, sendo encontrados dois itens complexos: 24 - Ter prestígio e 21 Ter fama. Pela teoria de valores pessoais de Schwartz (1992), é possível explicar esse fato relembrando que o valor Ter prestígio está associado ao tipo motivacional poder, e o valor Ter fama pode também estar relacionado ao tipo motivacional realização, sendo tais tipos adjacentes na estrutura motivacional, portanto possuem correlação. 


\section{TABELA 1. Resultados da análise fatorial da pesquisa}

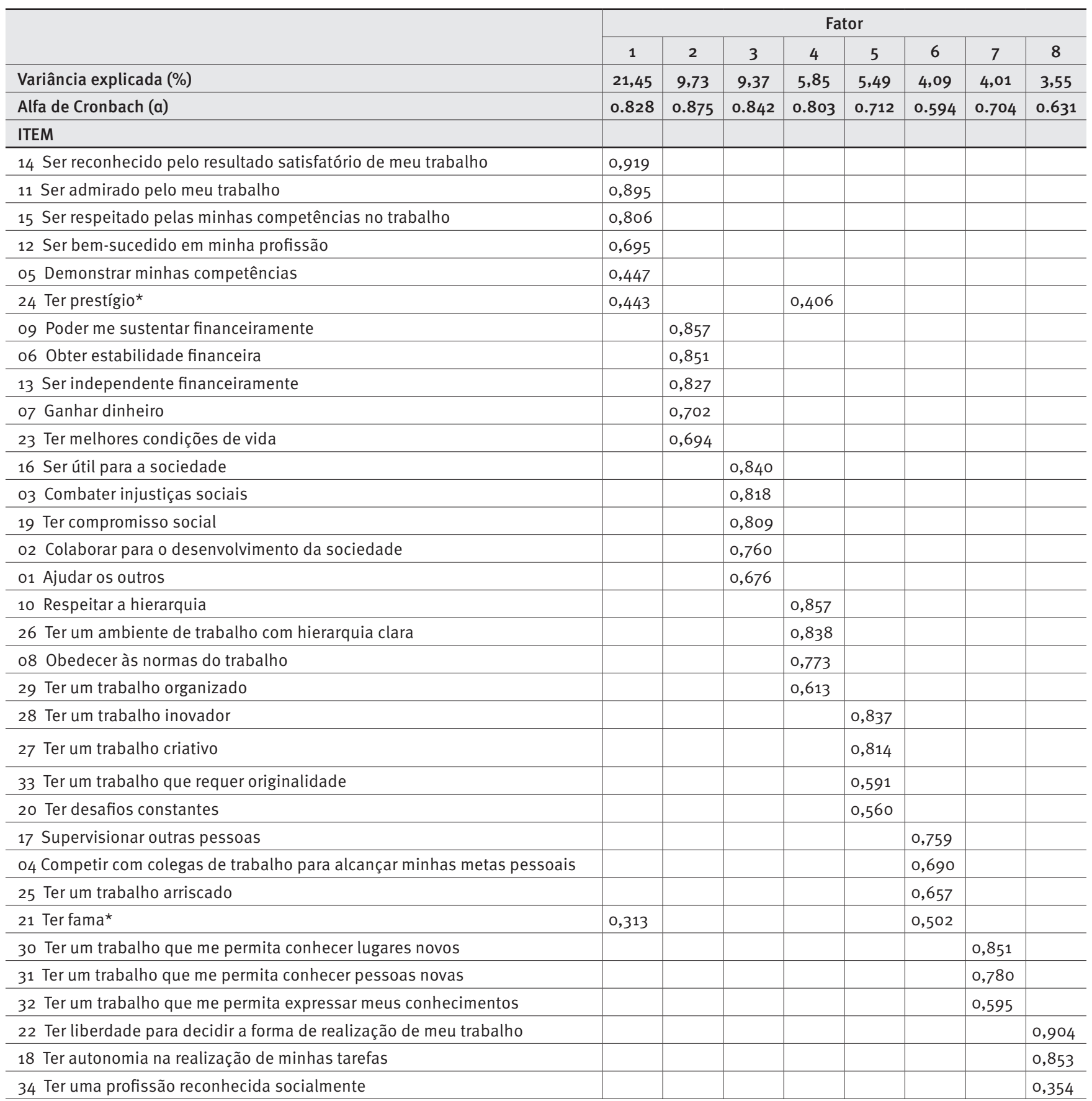

* Item complexo

Analisando os dados em comparação com a teoria utilizada, verificou-se que os itens componentes ao Fator 1 referemse ao tipo motivacional realização, visto que correspondem a valores relacionados à ambição, influência e inteligência. 0 Fator 2 foi considerado correspondente ao tipo motivacional segurança, representado por valores de estabilidade e ordem. 0 Fator 3 foi considerado correspondente aos tipos motivacionais universalismo e benevolência, relacionados à preocupa- ção com a sociedade, com a colaboração do grupo e com terceiros, em geral. 0 Fator 4 foi considerado correspondente ao tipo motivacional conformidade, configurado por itens relacionados a valores de autodisciplina e polidez. Os Fatores 5 e 7 foram considerados correspondentes ao tipo motivacional estimulação, relacionado a valores de inovação, desafios e mudança. 0 Fator 6 foi considerado correspondente ao tipo motivacional poder, representado pelos valores associados a poder 
social e autoridade. O Fator 8 foi considerado correspondente ao tipo motivacional autodeterminação, associado a valores de liberdade e criatividade.

Com relação ao estudo das prioridades axiológicas laborais da amostra estudada, foram calculadas as médias dos 7 fatores encontrados. Os resultados das médias indicaram que a ordem decrescente de valorização dos tipos motivacionais en- contrada para a amostra foi a seguinte: universalismo/benevolência, estimulação, segurança, autodeterminação, realização, conformidade, e poder. Os resultados encontrados por meio do cálculo das médias dos quatro conjuntos motivacionais indicaram a ordem decrescente de valorização a seguir: relações sociais, realização profissional, estabilidade e prestígio. Os dados estão resumidos na Tabela 2.

\section{TABELA 2. Prioridades axiológicas laborais da amostra estudada}

\begin{tabular}{l|c|c|c|l}
\hline Conjunto motivacional de segunda ordem & Média & Alfa de Cronbach & Fator & Tipos motivacionais referentes \\
\hline Relações Pessoais & 4,30 & 0,806 & 3 & Universalismo/Benevolência \\
\hline Realização Profissional & 3,90 & 0,842 & 5 & Estimulação \\
Realização Profissional & 3,90 & 0,842 & 7 & Estimulação \\
Realização Profissional & 3,90 & 0,842 & 8 & Autodeterminação \\
\hline Estabilidade & 3,85 & 0,838 & 2 & Segurança \\
Estabilidade & 3,85 & 0,838 & 4 & Conformidade \\
\hline Prestígio & 2,83 & 0,746 & 1 & Realização \\
Prestígio & 2,83 & 0,746 & 6 & Poder \\
\hline
\end{tabular}

\section{DISCUSSÃO}

Os resultados encontrados neste estudo permitem compreender a organização dos valores laborais da amostra estudada. $\mathrm{O}$ instrumento utilizado apontou para sete tipos motivacionais representados por oito fatores, conforme os agrupamentos da análise fatorial encontrados. Tais achados foram muito semelhantes aos resultados publicados por Porto e Pilati (2010), principalmente em relação aos agrupamentos dos Fatores 1, 2, 3,4 e 6 obtidos neste estudo. Os itens agruparam-se aqui, entretanto, em oito fatores, enquanto nos estudos dos autores da escala os itens agruparam-se em sete fatores. Analisando-se o conteúdo e itens desses agrupamentos, é possível considerar que os Fatores 5 e 7 correspondem ao mesmo tipo motivacional: estimulação, e, portanto, seriam obtidos, assim, sete fatores correspondentes, isto é, sete tipos motivacionais no total, conforme indicado pela EVT-R (Porto \& Pilati, 2010).

Segundo Porto e Pilati (2010), a EVT-R aproxima-se consideravelmente do modelo geral de valores e, por isso, possui representada a maioria dos tipos motivacionais, embora os tipos tradição e benevolência ainda estejam sub-representados na escala e deverão ser revistos em pesquisas posteriores. Não foram encontradas na literatura sobre valores relativos ao trabalho discussões referentes ao tipo motivacional hedonismo. Tais observações podem ser visualizadas nos resultados encontrados.
Analisando os fatores encontrados, é possível fazer algumas observações em relação a alguns itens agrupados. 0 item 24 - Ter prestígio, agrupado com maior carga ao Fator 1, poderia estar mais bem associado ao tipo motivacional poder, representado pelo Fator 6, o qual também foi associado, porém com menor carga fatorial. 0 item 34 - Ter uma profissão reconhecida socialmente, agrupado ao Fator 8, poderia estar mais bem associado ao tipo motivacional estimulação, representado pelos Fatores 5 e 7. Como já explicitado, entretanto, segundo a teoria de Schwartz (1992), considerando que se trata de tipos motivacionais adjacentes, estes podem apresentar correlação.

Analisando as médias para cada conjunto motivacional, foi possível verificar as prioridades axiológicas da amostra estudada. Os resultados permitem afirmar que os pesquisadores da organização de P\&D possuem conjunto motivacional predominante diferente do conjunto realização profissional. Tais resultados podem indicar a influência dos valores organizacionais da empresa estudada sobre os valores pessoais relativos ao trabalho dos pesquisadores que a ela pertencem. Pela caracterização da empresa, seus valores organizacionais visam ao desenvolvimento e inovação para o fortalecimento da investigação e para o benefício da sociedade como um todo, possuindo, portanto, um caráter altruísta. Os resultados de trabalho dos pesquisadores nessa organização devem, ainda, ser aplicáveis, propondo soluções sociais. Esses trabalhadores acabam, as- 
sim, por visar a resultados práticos, que beneficiem terceiros e a sociedade brasileira em geral, portanto valorizam fatores coletivos e altruístas.

É importante ressaltar que, embora o conjunto realização profissional não tenha sido o de maior média, ele se encontra em segundo lugar, confirmando seu alto grau de importância para a população estudada. Inovar e usar a criatividade, ter autonomia no trabalho e independência de pensamento, bem como buscar a realização pessoal, são também valores prioritários para os pesquisadores da organização analisada. Como indicado pela revisão da literatura, tais valores estão diretamente associados à inovação e ao trabalho de pesquisa em si, e são característicos da amostra deste estudo.

Visto que a organização estudada trata-se de uma empresa pública, que contrata os pesquisadores por meio de concurso público, e, considerando que esse tipo de contrato é visto como garantia de certa estabilidade em relação ao vínculo empregatício, é possível compreender a média alta encontrada para o tipo motivacional estabilidade. Tal fato indica que valores associados à busca de segurança e ordem na vida pessoal e profissional por meio do trabalho, com o objetivo de suprir necessidades pessoais por meio de bens materiais, isto é, de estabilidade financeira, também são considerados importantes pelos pesquisadores da amostra estudada.

O conjunto motivacional prestígio, que, pelo levantamento da literatura, poderia ser considerado com alto grau de importância por pesquisadores por representar valores relacionados à busca pelo sucesso profissional, reconhecimento e influência no ambiente de trabalho, obteve, neste estudo, a menor média. Essa evidência pode ser explicada ao se analisar que os valores associados a esse conjunto motivacional são antagônicos e conflitantes com o conjunto relações sociais. Visto que este último foi considerado o conjunto motivacional mais importante para os pesquisadores deste estudo, é, assim, condizente que seu conjunto antagônico, isto é, prestígio, não seja tão valorizado pela amostra estudada.

Os achados desta pesquisa permitem aprimorar o conhecimento sobre o que os pesquisadores buscam e valorizam em seus ambientes laborais, respondendo às perguntas de pesquisa e suprindo algumas lacunas encontradas sobre a gestão desse importante recurso humano, e também contribuindo com o conhecimento sobre comportamento organizacional. A maioria dos dados encontrados na revisão da literatura foi confirmada com os resultados deste estudo, por exemplo, a importância de alguns fatores levantados por Jordan (2005). Senso de desafio e entusiasmo, estilo de gestão informal, identificação de novas oportunidades, valorização a longo prazo da pesquisa fundamental podem ser associados ao tipo realização profissional.
Vojak et al. (2006) propuseram que os pesquisadores valorizam a criatividade e autonomia para tarefas, são profissionais idealistas, com amplitude e especialidade de conhecimento, emocionalmente envolvidos com o que fazem, sintetizando conhecimento e tecnologias a fim de alcançar novas descobertas. Todas essas características também foram enfatizadas nos resultados deste estudo, visto que as prioridades axiológicas mostram que os valores associados à realização profissional foram considerados importantes. A ideia de que os pesquisadores trabalham mais por mérito e reconhecimento do que por fator financeiro não foi, entretanto, intensificada com os resultados da pesquisa, sendo o conjunto prestígio o menos valorizado.

\section{CONSIDERAÇÕES FINAIS}

Inicialmente, pode-se concluir que o objetivo principal da pesquisa foi alcançado, visto que foram levantados e analisados os valores relativos ao trabalho de pesquisadores em uma organização pública de P\&D brasileira, definindo-se sua prioridade axiológica laboral.

O grande diferencial dos achados deste estudo remetese ao fato de que os pesquisadores apresentaram valores altruístas e coletivos, visando ao bem-estar do próximo, de terceiros e da sociedade em geral. Buscam fazer a diferença e realizar o que acreditam ser o certo, não por conta do mérito ou do reconhecimento primordialmente, mas porque objetivam contribuir com sua expertise e valorizam fazer algo útil e aplicado. Como já havia sido indicado por Katz (2005), esses profissionais sentem-se energizados quando consideram que estão fazendo algo importante, algo que realmente faça a diferença, nesse caso, para a sociedade brasileira.

Com os resultados, é possível compreender a importância dada à liberdade pelos pesquisadores no ambiente laboral e a necessidade de que eles mesmos definam seu próprio ritmo de trabalho e participem ativamente da construção de políticas e estratégias de negócios da empresa da qual fazem parte. Com isso, torna-se plausível o alinhamento dos valores desses profissionais com os valores organizacionais, possibilitando que melhores resultados de trabalho sejam alcançados. É necessário que haja harmonia entre os valores pessoais laborais desses trabalhadores com os valores da empresa, possibilitando o sentimento de produzir algo útil, que se considere importante.

Além dessas contribuições, este estudo também contribuiu com o conhecimento sobre a estrutura da escala utilizada, EVT-R, que foi aplicada em uma amostra homogênea e com características semelhantes. Os resultados das análises mostram que esse instrumento pode ser utilizado a fim de ampliar 
a compreensão de como os valores laborais afetam as escolhas e o comportamento organizacional, e permite uma avaliação mais completa sobre os valores laborais. Os achados também possibilitam enfatizar e confirmar a adequação da estrutura motivacional proposta por Schwartz (1992), sendo ela representativa dos valores pessoais no contexto do trabalho, bem como sua organização em conjuntos motivacionais de segunda ordem.

As principais limitações deste estudo estão relacionadas à amostra estudada. Por esta ter sido retirada de uma organização, por meio de aceite voluntário de vários pesquisadores, sendo as características destes não estritamente controladas, é considerada amostra não probabilística por conveniência. Cabe, assim, a discussão quanto à generalização dos resultados encontrados.

Além disso, por ter sido retirada de uma única organização, embora o objetivo tenha sido o controle da influência dos valores organizacionais, a fim de garantir características mais homogêneas, trata-se do estudo de um caso por meio de survey da uma determinada organização de $P \& D$ ou inovadora, não sendo oportuna a generalização dos resultados para outras organizações inovadoras.

Apesar das limitações, os resultados obtidos mostramse relevantes e interessantes para a literatura da área, e estudos futuros podem ser delineados a fim de minimizar tais limitações levantadas, bem como dar continuidade aos achados. Os resultados desta pesquisa emergem, ainda, como frutos de cuidadosa revisão da literatura referente à temática: valores relativos ao trabalho, e quanto ao objeto do estudo: pesquisadores em organização de $\mathrm{P} \& \mathrm{D}$, apresentando contribuições relevantes.

Conclui-se, assim, que os achados da pesquisa mostram a relevância de dar-se continuidade aos estudos relacionados aos valores laborais de pesquisadores, bem como aos estudos que foquem o ambiente de inovação. Merecem também atenção estudos futuros que contemplem questões envolvendo valores sociais, organizacionais e pessoais, com o intuito de compreender com maior afinco as influências sobre o comportamento organizacional em ambiente inovador; e estudos sobre valores laborais dos trabalhadores do conhecimento e a gestão da inovação. Com seus resultados, seria possível intensificar a contribuição em relação às informações sobre ferramentas de gestão organizacional e de recursos humanos nesses ambientes, possibilitando melhorias organizacionais. Informações teóricas e empíricas consistentes sobre o assunto podem, evidentemente, contribuir com a estratégia competitiva de inovação e, assim, promover melhores resultados de trabalho, tanto para a organização quanto para as pessoas que a compõem.

\section{REFERÊNCIAS}

Berson, Y, \& Linton, J. D. (2005). An examination of the relationships between leadership style, quality, and employee satisfaction in R\&D versus administrative environments. $R \& D$ Management, 35(1), 51-60.

Bohlander, G, Snell, S, \& Sherman, A. (2003). Administração de recursos humanos. São Paulo: Pioneira Thomson Learning.

Coda, R, \& Ricco, M. F. F. (2010). Estilos de comportamento de profissionais de ciência, tecnologia e inovação (C, T \& I). RAC-Revista de Administração Contemporânea, 14(3), 516-531.

Davenport, T. H. (2005). Thinking for a living, how to get better performance and results from knowledge workers. Boston: Harvard Business School Press.

Demo, G. (2008). Políticas de gestão de pessoas nas organizações: pa pel dos valores pessoais e da justiça organizacional. São Paulo: Atlas.

Drucker, P. (1999). Administrando em tempos de grandes mudanças. São Paulo: Pioneira.

Elizur, D, \& Sagie, A. (1999). Facets of personal values: a structural analysis of life and work values. Applied Psychology: an International Re view, $48(\mathrm{l}), 73-87$.

Forza, C. (2002). Survey research in operations management: a process based perspective. International Journal of Operations \& Production Management, 22(2), 152-194.

Gupta, A, \& Singhal, A. (1993). Managing human resources for innovation and creativity. Research Technology Management, 36(3), 41-48.

Hagström, T, \& Kjellberg, A. (2007). Stability and change in work values among male and female nurses and engineers. Scandinavian Journal of Psychology, 48(2), 143-151.

Hair Jr, J. F, Babin, B, Money, A. H, \& Samouel, P. (2005). Fundamentos de métodos de pesquisa em administração. Porto Alegre: Bookman.

Herzberg, F. (1971). Work and the nature of man. New York: World Publishing.

Jamrog, J, Vickers, M, \& Bear, D. (2006). Building and sustaining a culture that supports innovation. Human Resource Planning, 29(3), 9-19.

Jin, J, \& Rounds, J. (2012). Stability and change in work values: a meta-analysis of longitudinal studies. Journal of Vocational Behavior, $80(2), 326-339$.

Jordan, G. B. (2005). What matters to R\&D workers? Research Technology Management, 48(3), 23-32.

Katz, R. (2005). Motivating technical professionals today. Research Technology Management, 48(6), 29-27.

Lundberg, C, Gudmundson, A, \& Andersson, T. D. (2009). Herzberg's two-factor theory of work motivation tested empirically on seasonal workers in hospitality and tourism. Tourism Management, 30(6), 890899.

Maccoby, M. (2006). Is there a best way to lead scientists and engineers? Research Technology Management, 49(1), 60-61.

Miguel, L. A. P, \& Teixeira, M. L. M. (2009). Valores organizacionais e criação do conhecimento organizacional inovador. RAC-Revista de Administração Contemporânea, 13(1), 36-56.

Paarlberg, L. E, \& Perry, J. L. (2007). Values management: aligning employee values and organization goals. The American Review of Public Administration, 37(4), 387-408. 
Pereira, J. C. R, \& Saes, S. G. (1997). Definindo prioridades de gestão de ciência e tecnologia em saúde. Revista de Saúde Pública, 31(6), 624-631.

Porto, B. B, \& Pilati, R. (2010). Escala revisada de valores relativos ao trabalho - EVT-R. Psicologia: Reflexão e Crítica, 23(1), 73-82.

Porto, J. B, \& Tamayo, A. (2003). Escala de valores relativos ao trabalho - EVT. Psicologia: Teoria e Pesquisa, 19(2), 145-152.

Porto, J. B, \& Tamayo, A. (2007). Estrutura dos valores pessoais: a relação entre valores gerais e laborais. Psicologia: Teoria e Pesquisa, 23(1), 63-70.

Porto, J. B, \& Tamayo, A. (2008). Valores do trabalho. In: M. M. M. Siqueira (Org.). Medidas do comportamento organizacional: ferramentas de diagnóstico e de gestão. Porto Alegre: Artmed.

Ros, M, Schwartz, S. H, \& Surkiss, S. (1999). Basic individual values, work values, and the meaning of work. Applied Psychology: An International Review, 48(1), 49-71.

Sagie, A, \& Elizur, D. (1996). The structure of personal values: a conical representation of multiple life areas. Journal of Organizational Behavior, $17(\mathrm{~S} 1), 573-586$.

Schwartz, S. H. (1992). Universals in the content and structure of values: theoretical advances and empirical tests in 20 countries. In M. P. Zanna (Ed.). Advances in experimental social psychology (Vol. 24, pp. 1-65). San Diego: Academic.

Schwartz, S. H. (1999). A theory of cultural values and some implications for work. Applied Psychology: An International Review, 48(1), 2347, 1999.
Schwartz, S. H. (2005). Valores humanos básicos: seu contexto e estrutura intercultural. In A. Tamayo, \& J. B. Porto (Orgs.). Valores e comportamento nas organizações. Petrópolis: Vozes.

Schwartz, S. H. (2011). Studying values: personal adventure, future directions. Journal of Cross-Cultural Psychology, 42(2), 307-319.

Silva, P. G, \& Ricco, M. F. F. (1998). Gestão de P\&D no centro técnico aeroespacial: normas como ferramentas. Anais do Simpósio da Gestão da Inovação Tecnológica, São Paulo, 20.

Super, D. E. (1953). A theory of vocational development. American Psychologist, 8(4), 185-190.

Tamayo, A. (2007a). Contribuições ao estudo dos valores pessoais, laborais e organizacionais. Psicologia: Teoria e Pesquisa, 23(n. especial), 17-24.

Tamayo, A. (2007b). Hierarquia de valores transculturais e brasileiros. Psicologia: Teoria e Pesquisa, 23(especial), 7-15.

Tamayo, A., Faria, J. B., Filho, A. B., Tavares, M. S. O. C., Carvalho, E. M., \& Bertolinni, V. (1998). Diferenças nas prioridades axiológicas de músicos e advogados. Psicologia: Reflexão e Crítica, 11(2), 281-294.

Tamayo, A, \& Paschoal, T. (2003). A relação da motivação para o trabalho com as metas do trabalhador. RAC-Revista de Administração Contemporânea, 7(4), 33-54.

Verdu, F. C, \& Bulgacov, S. (2001). Redes de pesquisadores da área de administração: um estudo exploratório. RAC-Revista de Administração Contemporânea, ed. especial, 163-182.

Vojak, B. A, Griffin, A, Price, R. L, \& Perlov, K. (2006). Characteristics of technical visionaries as perceived by American and British industrial physicists. R\&D Management, 36(1), 17-26. 